\title{
LQG Control of TCSC for Damping Sub Synchronous Resonance
}

\author{
Basim Talib \\ Electrical Engineering \\ University of Basra/ Iraq
}

\author{
Khalid Abdulsadaa \\ Electrical Engineering \\ University of Basra/ Iraq
}

\begin{abstract}
This research shows that when connecting a generator and steam turbines to a transmission line that has series capacitive compensation, this may cause many problems, including the emergence of a sub-synchronous resonance state. The Flexible Ac Transmission System (FACTS) controller of the devices wide use which has been used to reduce and suppress the phenomenon of sub-synchronous resonance the Thyristor Control Series Compensation (TCSC) is one type of FACTS controller that has been used to put down the SSR. The first standard model of IEEE was adopted to study this phenomenon. Linear Quadratic Gaussian (LQG) was used to design TCSC strong to dampen this phenomenon in the energy system. Reduce- Order version of this controller is also obtained. Non-linear systems simulations are used to achieve a better control unit to dampen all oscillations in a very short time. In this paper, we also examine the process of controlling the parameters of TCSC and AVR through the use of PID controller To achieve a process of suppression of this phenomenon. In this research, the method of Eigenvalue was used to analyze the sub-synchronous resonance phenomenon and the result was verified using DYMOLA simulation.
\end{abstract}

\section{General Terms}

Sub Synchronous Resonance, Kalman filter, TCSC, state space.

\section{Keywords}

Flexible AC Transmission Systems (FACTS), Linear Quadratic Gaussian (LQG), TCSC (Thyristor Control Series Compensation), SSR (Sub -Synchronous Resonance), Kalman filter, power system oscillation damping.

\section{INTRODUCTION}

The series compensation are to large extent used to increase the transmission of energy in the transmission lines and increase the stability of the transient situation as well as to play a role in the process of load sharing in parallel transmission lines[1]. These functions are accomplished by partial compensation of the transmitter line reactor. Despite these benefits, the compensation chain may cause many problems, including the emergence of a condition called subsynchronous resonance in the shaft of turbine-generator with fluctuations in system frequencies. When the normal frequency of the system is equal to one of the alternating frequencies of the alternator shaft and turbine, in this case we have a phenomenon called sub-synchronous resonance [2], SSR which happens when energy is exchanged between the generator of the turbine and the electrical network in case of one or more frequencies of the system with frequencies below Synchronous system. When the rotor oscillation it will build a small voltage this voltage will generate an asynchronous current. This current will produce oscillating torque which can cause oscillations in the rotor, which will cause significant damage to the turbine shaft. [4] [5]. There are many modern methods that have been used to avoid the problem of SSR by using appropriate systems to avoid the sub synchronous voltage and the current, has been using Flexible Ac Transmission System (FACTS) and various types TCSC, SVC, SSSC, STATCOM to mitigate the damage that falls on the turbine shaft as well as the treatment of sub-synchronous resonance [6]. In the modern power system it can be used Thyristor Control Series Compensation (TCSC) in an increasingly sophisticated way as (TCSC) allows changing the transmission line impedance quickly and continuously [7]. In The power system the TCSC provides several important advantages including sub-synchronous resonance suppression as well as control of the energy flow process in the transmission line [8].This research deals with the damping and control of the luminescent oscillations generated by T-G through the use of the controller Linear Quadratic Gaussian (LQG) the ability of this type of controller to provide a good performance to put down the oscillations generated and also can be carried out a reduction in the control unit designed. Linear-Quadratic-Gaussian (LQG) control is commonly applied for these control purposes. The LQG consists of a general from Kalman filter and a Linear-Quadratic Regulator (LQR).LQG optimal control techniques have been applied in many linear dynamic system cases since 1960s [9- 11]. An optimal LQG controller for linear stochastic systems with unknown parameters has been developed by Basin et al. "The original controller problem was split into the optimal filtering problem for incompletely measured bilinear states over linear observations and the optimal control (regulator) problem for the designed filter estimate" [12]. Petersen et al reported a practical robust controller design methodology based on a mini max LQG control for the control of vibrations in a flexible cantilever beam [13]. In this research we will explain the effect of sub-synchronous resonance condition as well as the mechanism used to extinguish this phenomenon by using the TCSC [14], as well as the mechanism used to control the parameters of TCSC once using PID control and other using (LQG).

\section{SYSTEM MODEL}

\subsection{Power System}

The studied system is the First IEEE Benchmark Model [15], for SSR analysis, depicted in Fig. 1. An 892.4 MVA synchronous generator is connected to an infinite bus via a highly compensated $500 \mathrm{kV}$ transmission line. The mechanical system consists of a four-stage steam turbine, the generator and a rotating exciter. The electrical and mechanical systems were modeled using the DYMOLA program the generator was represented by a Type-59 synchronous machine source component. Two damper windings were provided in the axis while in the axis one damper and a field winding were considered. The mechanical system was represented by a multi-mass spring-dashpot system, with six lumped masses 
coupled by shaft sections of known torsional elasticity. Mechanical damping was assumed to be zero in all the analyzed cases, to represent the worst damping conditions. The complete electrical and mechanical data for the studied system are presented in reference [15].

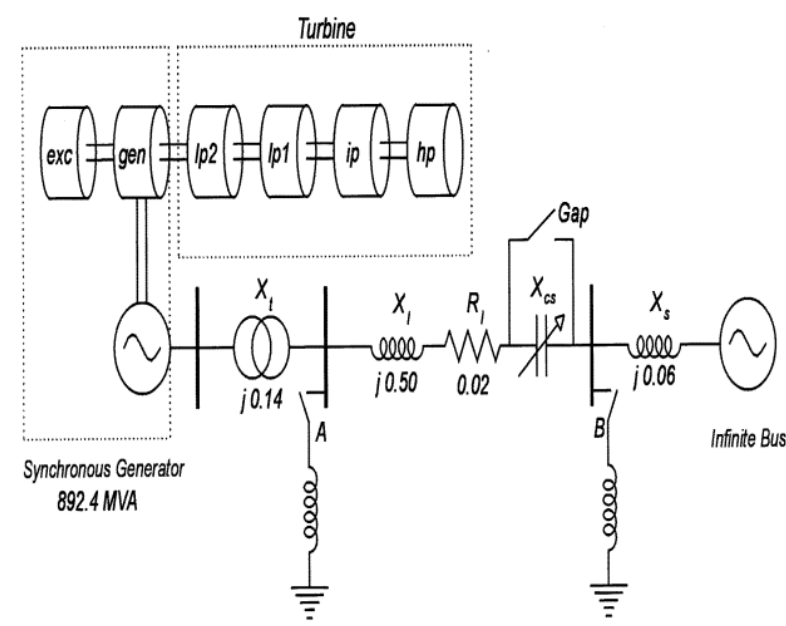

Figure 1: Mathematical Model

\subsection{Excitation System Model}

The system uses the excitement of processing direct current files to the field of synchronous generator. As well as by controlling the field voltage Efd and the current field provides a control mode in the stable energy system. In this search system the user is excited IEEE STA1 type on automatic excitation regulator shown in Fig.2.

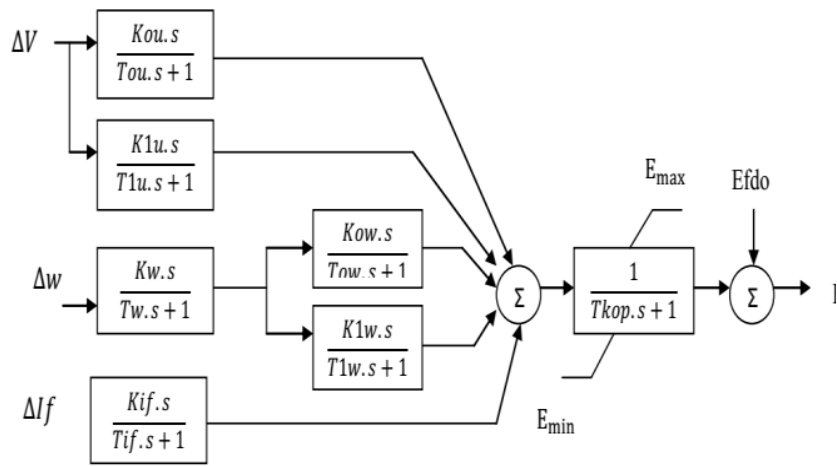

Figure 2: Modeling of Excitation System

\section{ANALYSIS oF SS}

Under the conditions below, the analysis of SSR was performed

1.The transportation system receives a sum of 1 p.u from the generator.

2. The turbine energy inside it is assumed fixed.

in this paper the method of analysies or damping of the SSR can by divided into three parts:
1. Damping without control of TCSC
2. Damping of SSR with control of TCSC and AVR
3. Damping of SSR with optimal control(LQG)

\subsection{Damping without Control of TCSC}

In the first studied, by changing the level compensation from $(0 \%$ to $100 \%)$ we observe that the first IEEE standard system will have four unstable frequencies. These unstable frequencies increase the phenomenon of sub synchronous resonance [16]. Without the control of TCSC and AVR also, at different levels of compensation, we observe that torsional frequencies have unstable roots, note that four are different levels of compensation magnitude. Table 1 , shows the value of real part for each frequency without any damping and using Eigen value analytical.

Table 1: First Benchmark Eigenvalue without any Control

\begin{tabular}{|c|l|l|l|l|}
\hline mode & \multicolumn{4}{|c|}{ \%Compensation Level } \\
\hline & $21.41 \%$ & \multicolumn{1}{|c|}{$39.28 \%$} & $57.41 \%$ & $75 \%$ \\
\hline 0 & $-0.24 \mp 27.7 \mathrm{i}$ & $-0.1 \mp 88.6 \mathrm{i}$ & $-0.2 \bar{\mp} 29.28 \mathrm{i}$ & $-0.29 \mp 10.14 \mathrm{i}$ \\
\hline 1 & $-0.03 \mp 298 \mathrm{i}$ & $-0.0184 \bar{\mp} 98 \mathrm{i}$ & $0.041 \mp 698 \mathrm{i}$ & $2.6 \bar{\mp} 898 \mathrm{i}$ \\
\hline 2 & $0.03 \mp 9127 \mathrm{i}-$ & $-0.03 \bar{\mp} 6127 \mathrm{i}$ & $0.240 \bar{\mp} 127 \mathrm{i}$ & $-0.03 \bar{\mp} 9127 \mathrm{i}$ \\
\hline 3 & $-0.06 \bar{\mp} 7161 \mathrm{i}$ & $0.60 \bar{\mp} 5161 \mathrm{i}$ & $0.077 \bar{\mp} 161 \mathrm{i}-$ & $-0.07 \bar{\mp} 9161 \mathrm{i}$ \\
\hline 4 & $0.76 \bar{\mp} 0203 \mathrm{i}$ & $-0.022 \bar{\mp} 7203 \mathrm{i}$ & $-0.02 \bar{\mp} 5203 \mathrm{i}$ & $-0.02 \bar{\mp} 3203 \mathrm{i}$ \\
\hline 5 & $0.1 \bar{\mp} 0299 \mathrm{i}-$ & $0 . \bar{\mp} 1299 \mathrm{i}-$ & $0 . \bar{\mp} 1299 \mathrm{i}-$ & $0.1 \bar{\mp} 299 \mathrm{i}-$ \\
\hline $\begin{array}{r}\text { Ele.m } \\
\text { od }\end{array}$ & $-4.63 \bar{\mp} 203 \mathrm{i}$ & $-4.2 \bar{\mp} 161 \mathrm{i}$ & $-3.5 \bar{\mp} 2127 \mathrm{i}$ & $-5.21 \bar{\mp} 98 \mathrm{i}$ \\
& & & & \\
\hline
\end{tabular}

\subsection{Damping of SSR with Control of TCSC}

The TCSC can be controlling by using a proportionalintegral- derivative (PID) controller. Many theoretical analysis\& field tests of [17], the torsional oscillations are triggered by the use of a rotor signal to create a firing angle. The modulation firing angle has few effects on SSR. In TCSC use it to indicate the voltage of the line as well as the speed signal of the generator as a feedback for control the Eigen value, Table 2 . Shows the values of the torsional mode and the critical levels when controlling the use of the voltage signal as well as the speed indication to control the parameters of TCSC. The equivalents modeling of TCSC can be represented in Fig.3. The line voltage input into TCSC across fixed capacitor error $\Delta U c$. The signal auxiliary is the speed deviation $\Delta W C$ is add to $\Delta U C$ for reduce the inertial and torsion oscillation modes.

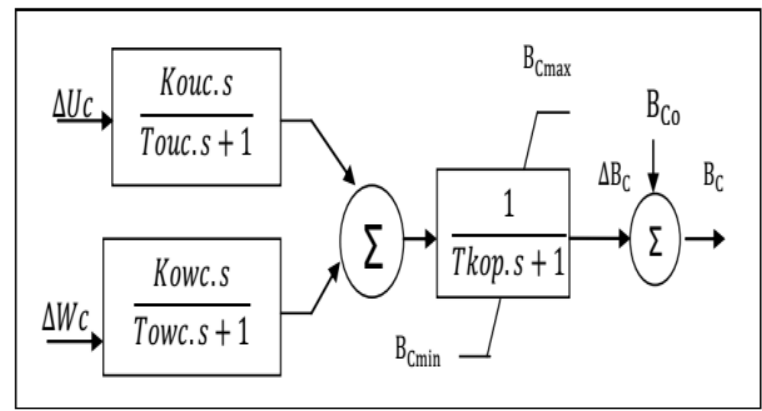

Figure 3: Modeling of TCSC

All the control channels specified in this section are selected on the basis of obtaining maximum damping at the frequencies of torsional oscillations. Where $K_{\text {ouc }}$ is the voltage regulation; $T_{\text {ouc }}$ is the time constant of the channel and $K_{o w c}$ is the gain of the frequency deviation channel and $T_{\text {owc }}$ is the time constant of the frequency deviation channel. Rate constants of time -mentioned above selected to give the best values to real Eigen value while the value of $B_{c m i n}>0$ and $B_{c \max }<100 \%$ level compensation the effected by this. The technique eigenvalue was used to 
analyze the stability of the system and was observed at the specified point of operation showing its ability TCSC to stabilize the torsional oscillations.

Table 2:First Benchmark Eigenvalues with TCSC and AVR Control

\begin{tabular}{|l|l|l|l|l|}
\hline Mode & \multicolumn{4}{|c|}{ \%Compensation Level } \\
\hline & $21.42 \%$ & $39.28 \%$ & $57.41 \%$ & $75 \%$ \\
\hline 0 & $-0.821 \bar{\mp} 6.52 i$ & $-0.928 \bar{\mp} 7.22 i$ & $1.0 \bar{\mp} 88.57 i-$ & $-1.24 \bar{\mp} 9.16 i$ \\
\hline 1 & $-0.073 \mp 98 i$ & $-0.078 \mp 98 i$ & $-0.07 \mp 798 i$ & $-0.010 \bar{\mp} 298 i$ \\
\hline 2 & $-0.058 \bar{\mp} 127 i$ & $-0.05 \bar{\mp} 8127 i$ & $0.04 \bar{\mp} 8127 i-$ & $-0.03 \bar{\mp} 4127 i$ \\
\hline 3 & $0.094 \bar{\mp} 161 i-$ & $0.08 \bar{\mp} 7161-$ & $-0.05 \bar{\mp} 0161 i$ & $-0.07 \bar{\mp} 8161 i$ \\
\hline 4 & $0.09 \bar{\mp} 1203 i-$ & $0.00 \bar{\mp} 2203 i-$ & $-0.23 \bar{\mp} 3203 i$ & $-0.02 \bar{\mp} 2203 i$ \\
\hline 5 & $0.10 \bar{\mp} 299-$ & $0.1 \bar{\mp} 0299 i-$ & $-0.1 \bar{\mp} 0299 i$ & $-0.1 \bar{\mp} 0299 i$ \\
\hline $\begin{array}{l}\text { Ele. } \\
\text { Mode }\end{array}$ & $-0.02 \bar{\mp} 6214 i$ & $-1.7 \bar{\mp} 6174 i$ & $-2.4 \bar{\mp} 3141 i$ & $-1.9 \bar{\mp} 2112 i$ \\
\hline
\end{tabular}

\subsection{Damping of SSR with Optimal Control (LQG)}

LQG is used in many applications ranging from aviation and missile as well as control systems in navigation and nuclear stations as well as in medical operations. It is generally composed of Kalman filters and also from the Linear Quadratic Regulator (LQR) for full stat feedback. The state space equations of the open loop plant for a standard LQG problem is shown in eq. 1 .

$$
\begin{aligned}
& x \dot{x(t)}=A x(\mathrm{t})+B u(t)+G w(t) \\
& y(t)=C x(\mathrm{t})+v(t)
\end{aligned}
$$

where $x(t)$ is the state vector, $u(t)$ is the control input vector, $y(t)$ is the measured output vector, $w(t)$ and $v(t)$ are stochastic white noise processes assoctiated with the process and the measurement, respectively. The matrices $\mathrm{A}$ (state matrix), $\mathrm{B}$ (control input gain matrix), $\mathrm{G}$ (plant noise gain matrix), $\mathrm{C}$ (measured state matrix) are all Linear Time Invariant (LTI). As shown in eq.2. Both $w(t)$ and $v(t)$ are assumed to be white gaussian noises with zero mean and the expected values of the initial values of the states $x(0)$ are assumed to be equal to $\tilde{x}_{o}$. From eq.3. $w(t)$ and $v(t)$ have covariance matrices of $\mathrm{W}$ and $\mathrm{V}$, respectively, and the cross covariance $R 12$ is assumed to be zero ( $w(t)$ and $v(t)$ are uncorrelated) in order to simplify many expressions and derivations. Finally, the initial values of the states $x(0)$ are assumed to be uncorrelated with both $w(t)$ and $v(t)$, as shown in eq. 4 .

$$
\begin{array}{cc} 
& E[x(0)]=\tilde{x}_{o} \\
E[w(t)]=0 &
\end{array}
$$

$$
\begin{aligned}
E\left[w(t) w(\tau)^{\mathrm{T}}\right] & = \begin{cases}W & \text { if } t=\tau \\
0 & \text { if } t \neq \tau\end{cases} \\
E\left[v(t) v(\tau)^{\mathrm{T}}\right] & = \begin{cases}V & \text { if } t=\tau \\
0 & \text { if } t \neq \tau\end{cases}
\end{aligned}
$$$$
E\left[w(t) v(\tau)^{\mathrm{T}}\right]= \begin{cases}R_{12} & \text { if } t=\tau \\ 0 & \text { if } t \neq \tau\end{cases}
$$

$$
\begin{gathered}
E\left[v^{\mathrm{T}}(t) x(t)\right]=0 \\
E\left[w^{\mathrm{T}}(t) x(t)\right]=0
\end{gathered}
$$

One of the problems of control is that it is not possible to get all the state of the system which are necessary to get full reactions to the state, instead of getting all the outputs of the system we will get specific outputs of the system to carry information about the state of the state space equations of the Kalman Filter are shown in eq.5. So the same state matrices can be used (A, B and C) also the estimated states and the main system $(\tilde{x}(t))$ are used as the system states [19].

$$
\tilde{x}=\left(A-K_{k} C\right) \tilde{x}(t)+B u(t)+K_{k} y(t)
$$

$\tilde{y}(t)=C \tilde{x}(t)$

Through Fig.4. We find that LQG can be configured by connecting the system to the Kalman filter throught the optimal state estimation gain $\left(K_{k}\right)$ and then creating full state feedback by using the estimated states $(\tilde{x}(t))$ which passed through the optimal feedback gain $\left(k_{f}\right)$.The first step of the design starts by checking the observability and controllablity pairs (A, B) and (A, C), respectively, as shown in eq.6 These standards are important in the process of obtaining optimal gains in equation solutions.. Then, the optimal state estimation gain $\left.\left(K_{k}\right)\right)$ is calculated as shown in eq(7c), where $\left(P_{k}\right)$ is a positive semi-definite mtarix and the solution of the Filter Algebraic Riccati Equation (FARE) shown in eq(7b). This solution ensures a minimum value of the cost function shown in eq.7a. After that, the optimal state feedback gain $\left(K_{f}\right)$ ) is calculated as shown in eq.8c. Where $P f$ is a positive semidefinite matrix and the solution of the Control Algebraic Riccati Equation (CARE) shown in eq(8b). The lowest cost can be obtained through this solution shown in eq.8a. Unlike FARE, which requires only noise covariances $\mathrm{W}$ and $\mathrm{V}$, CARE requires two weighing matrices: $Q_{f}$ (symmetric positive semi-definite) and $R_{f}$ (symmetric positive definite). Using the trial and error mode you can choose matrix weights or by using the method shown in eq.9. Or by using Bryson's Rule shown in eq.10. Bryson's Rule related the reciprocal of the maximum squared values of the states with $Q_{f}$ and the reciprocal of the maximum squared values of the control inputs with $R_{f}$. After finding the optimal gains, the closed loop system could be created by augmenting the system with Kalman Filter resulting in the new model shown in eq.11. The augmented system model which include the dynamics of the system and the state estimation error $e(t)$.

Step 1: Calibrate the existence of optimal gain:

$(A ; B)$ is Controllable

$(A ; C)$ is Observable

Step 2: Account gain of the Optimal State Estimation:

$$
\begin{aligned}
& J_{k}=E\left\{(x-\tilde{x})^{\mathrm{T}}(x-\tilde{x})\right\} \\
& A P_{k}+A^{\mathrm{T}} P_{k}+G W G^{\mathrm{T}}-C^{\mathrm{T}} P_{k} V^{-1} C P_{k}=0 \\
& K_{K}=C^{\mathrm{T}} P_{k} V^{-1}
\end{aligned}
$$

Step 3: Choose weights of matrices as well as calculate gain optimal State Feedback

$$
\begin{gathered}
J_{f}=\int_{0}^{\mathrm{T}}\left(z^{\mathrm{T}} Q_{f} z+u^{\mathrm{T}} R_{f} u\right) d t \\
A P_{f}+A^{\mathrm{T}} P_{f}+Q_{f}-P_{f} B R_{f}^{-1} B^{\mathrm{T}} P_{f}=0
\end{gathered}
$$




$$
\begin{aligned}
K_{f} & =B^{\mathrm{T}} P_{f} R_{f}^{-1} \\
Q & =C^{\mathrm{T}} C \\
R & =\rho I
\end{aligned}
$$

$Q_{i i}$

$$
=\frac{1}{\operatorname{Max}\left(x_{i i}\right)}
$$$$
R_{i i}=\frac{1}{\operatorname{Max}\left(u_{i i}\right)}
$$

Step 4: LQG consists of Optimal State Feedback and Optimal State Estimation:

$$
\begin{aligned}
& {\left[\begin{array}{l}
x^{\cdot}(t) \\
e^{\cdot}(t)
\end{array}\right]=\left[\begin{array}{cc}
A-B K_{f} & B K_{f} \\
0 & A-C K_{k}
\end{array}\right]\left[\begin{array}{l}
x(t) \\
e(t)
\end{array}\right]+\left[\begin{array}{cc}
G & 0 \\
G & -K_{k}
\end{array}\right]\left[\begin{array}{l}
W(t) \\
V(t)
\end{array}\right]} \\
& y(t)=\left[\begin{array}{ll}
C & 0
\end{array}\right]\left[\begin{array}{l}
x(t) \\
e(t)
\end{array}\right]+\left[\begin{array}{ll}
0 & 1
\end{array}\right]\left[\begin{array}{l}
W(t) \\
V(t)
\end{array}\right]
\end{aligned}
$$

Table 3:Eigenvalues of Close Loop for TCSC Regulator

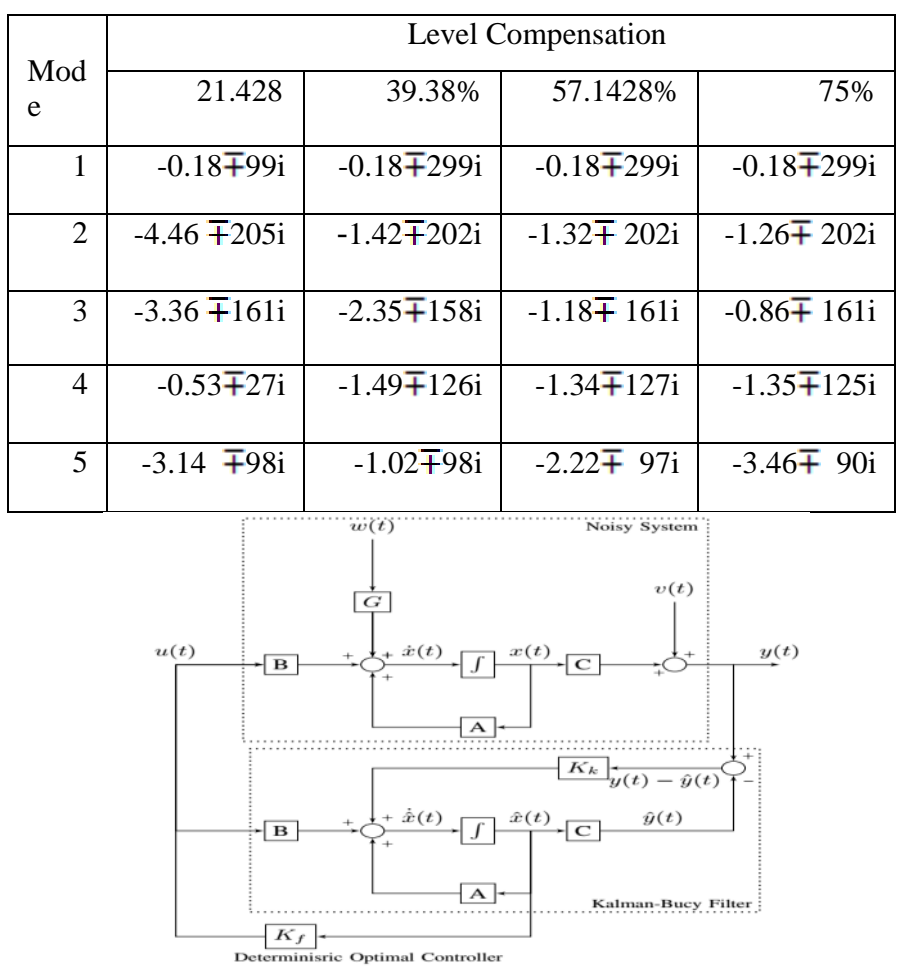

Figure (4) LQG Regulator Block Digram

Figure below display the control system used to control the TCSC. And its main input signal is signal of voltage deviation on the buses of the device $U_{C}$ (without derivative), and signal of speed deviation $W_{C}$ also the signal of line current deviation $I_{C}$. After the specified signals are used to control of TCSC these signals the outside of the system $G(s)$ will return as feedback each signal will enter the block of the $H(s)$, the block of $H(s)$ consist from the transfer function have the same order of the $G(s)$. The signals outside the block of the $H(s)$ will be compared with the input signals to obtain a minimum error ratio.

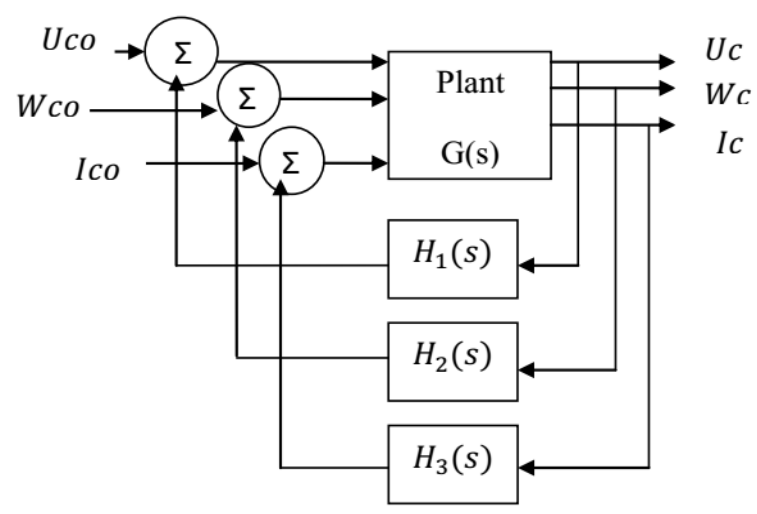

Figure 5: Block Diagram of the Control System of the TCSC with Optimal Control

In Table 3.Shows the results of calculations of the damping parameters of the model with various control systems. From this table it can be seen that the developed robust stabilizer at $\left(26^{\mathrm{t}} \mathrm{h}\right)$ provides a significant improvement in the damping properties of the system at torsional vibration frequencies. In this paper we will explain the effect of Linear Quadratic Gaussian (LQG) on putting out the sub synchronous resonance (SSR) state in the system by performing TCSC control only without the control function of the AVR only to perform a control operation of the network part. In the TCSC control process using three signals (voltage and current and speed deviation as shown in Fig.5. Enable it to obtain better results than control mode using PID as well as with better stability and for all cases of change in the level compensation. This results obtained in calculations of the static stability.

\section{MODEL ORDER REDUCTION}

For the purpose of obtaining an easy control process, a process of reducing the complex systems is done through the use of techniques to reduce the model. Reducing the system provides some important advantages, for example. Determine the critical characteristics of the system as well as eliminate the system modes that are not related to the control. The process of using a lot of modern control methodologies based on the controller (LQG, Hळ) has led to the use of the technique of reducing the model. The Hankel-Normative approach is one of the most important developments in the mechanism of reducing the model because of the narrow limits of error on the base of determinism. There are many forms of this technique (balanced Truncation, Hankel-norm optimal, relative-error), [20], these algorithms are used effectively in small and medium systems while in the case of large systems and very complex algorithm is used (Krylov Subspace),[21].The mathematical formulation of the problem consists in obtaining a low order approximation $\mathrm{Gr}(s)$ for a given linear system of high order $\mathrm{G}$ (s) such that the infinite norm their distinction $\| \mathrm{G}$ - Gr $\| \infty$ is minimal. It is necessary to calculate a model of a reduced order:

$$
\mathrm{G}_{\mathrm{r}}(\mathrm{s})=\mathrm{G}_{\mathrm{r}}\left(\mathrm{sI}-\mathrm{A}_{\mathrm{r}}\right)^{-1} \mathrm{~B}_{\mathrm{r}}+\mathrm{D}_{\mathrm{r}}
$$

From the complete $\mathrm{n}^{\text {th }}$ full system drop model

$$
G(s)=C(s
$$

Such that 
Whereas $\sigma_{\mathrm{i}}$ represent the value of Hankel singular to the plant $G(j w)$, while the value of square roots to the controllability and observability grammars:

$$
\sigma_{\mathrm{i}}=\sqrt{\lambda_{\mathrm{i}}(P Q)}
$$

P and $Q$ values are used to solve equations Lyapunov;

$$
\begin{aligned}
A^{\mathrm{T}}+A P+B B^{\mathrm{T}}= & 0 \text { (contollablity grammian) }-- \\
& -(16)
\end{aligned}
$$

$Q A+A^{\mathrm{T}} Q$

Not that A, B, C, D is the state space matrices of the full order model $G(s)$ while $A_{r}, B_{r}, C_{r}$ and $D_{r}$, are analogous matrices of a system of reduced order Gr (s). Table 4. Present the perfect eigenvalues for the close-loop adapted from the reduced order reduction at level compensation $21.4285 \%$.

\begin{tabular}{|c|c|c|c|c|}
\hline $\begin{array}{l}\text { No. of } \\
\text { Reduction }\end{array}$ & $\begin{array}{r}203 \\
\mathrm{red} / \mathrm{sec}\end{array}$ & $161 \mathrm{red} / \mathrm{sec}$ & $\begin{array}{r}127 \\
\mathrm{red} / \mathrm{sec}\end{array}$ & $\begin{array}{r}98 \\
\mathrm{red} / \mathrm{sec}\end{array}$ \\
\hline $26^{\text {th }}$ & $-4.46 \mp 203 i$ & $-3.36 \mp 161 i$ & $-0.53 \mp 127 \mathrm{i}$ & $-3.14 \mp 98 \mathrm{i}$ \\
\hline $20^{\text {th }}$ & $-4.36 \bar{\mp} 203 \mathrm{i}$ & $-3.23 \bar{\mp} 161 \mathrm{i}$ & $-0.36 \mp 127 i$ & $-3.12 \bar{\mp} 98 \mathrm{i}$ \\
\hline $12^{\text {th }}$ & $-3.32 \bar{\mp} 203 \mathrm{i}$ & $-2.87 \bar{\mp} 161 \mathrm{i}$ & $-0.34 \mp 127 i$ & $-2.78 \mp 98 \mathrm{i}$ \\
\hline $10^{\text {th }}$ & $-1.8 \bar{\mp} 203 \mathrm{i}$ & $-0.25 \bar{\mp} 161 \mathrm{i}$ & $-0.34 \bar{\mp} 127 i$ & $-0.27 \bar{\mp} 98 \mathrm{i}$ \\
\hline $\mathrm{r} 6^{\text {th }}$ & $-1.81 \bar{\mp} 203 \mathrm{i}$ & $-0.19 \bar{\mp} 161 \mathrm{i}$ & $-0.34 \bar{\mp} 127 \mathrm{i}$ & $-0.25 \mp 98 \mathrm{i}$ \\
\hline
\end{tabular}

Table(4) Eigenvalues of Closed loop for Regulation of Different Order

\section{DYNAMIC RESULT}

Fig.6. Below shows that system response for three phase short circuit at $0.01 \mathrm{sec}$ and level compensation $21.428 \%$. Fig.6a. Show the electromechanical torque (Me1) Fig.6b Shows curves of torque between the low pressure turbine and generator $(\mathrm{M} 1 \mathrm{~g})$ with full order LQG controller for $12^{\mathrm{th}}$ and Fig.6c. Show the terminal voltage of generator $(\mathrm{Ug})$ and Fig.6d. Show the speed devotion of generator. Fig.7a. Shows the torque between the low pressure turbine and the generator (LPA-GEN) with reduce order LQG controller to $6^{\mathrm{t}} \mathrm{h}$ and Fig.7b. Show the terminal voltage of generator with reduce order LQG controller to $6^{\mathrm{t}} \mathrm{h}$ and Fig.7c. Show the electromechanical torque. All figures at level compensation $21.248 \%$.

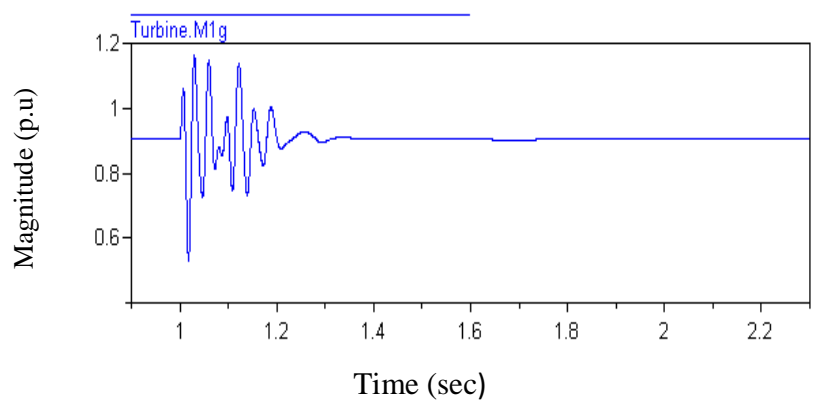

Figure6a: Torque between the LPA-GEN

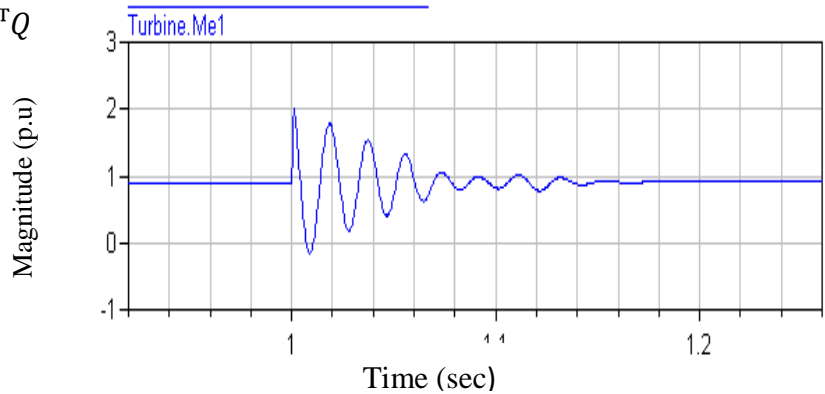

Figure6b: Electromechanical Torque (Me1)

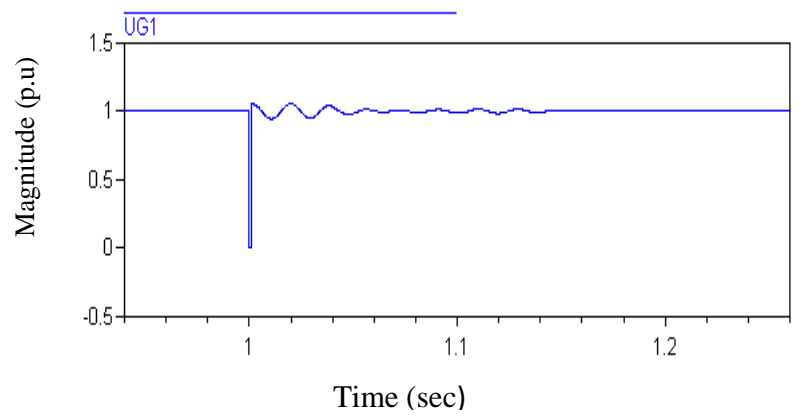

Figure 6c: Terminal Voltage of Generator

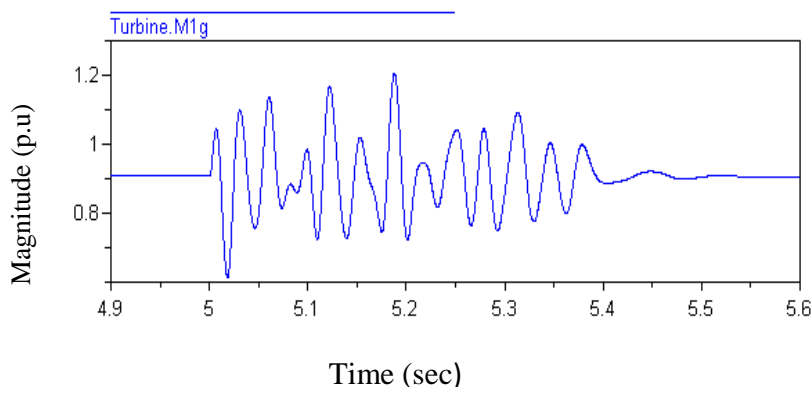

Figure 7a: Torque between LPA-GEN

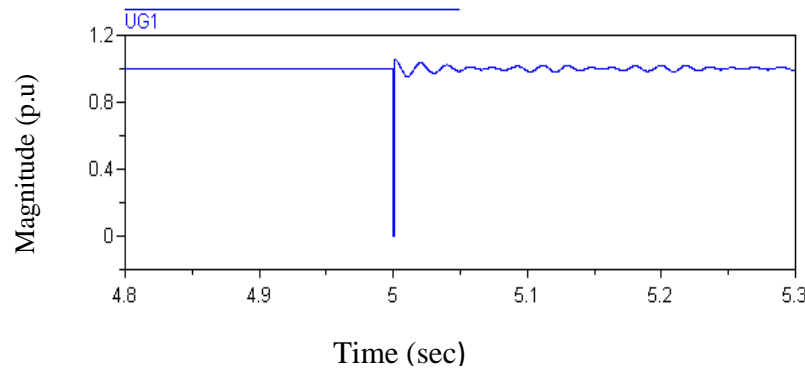

Figure 7b: Terminal Voltage of Generator 


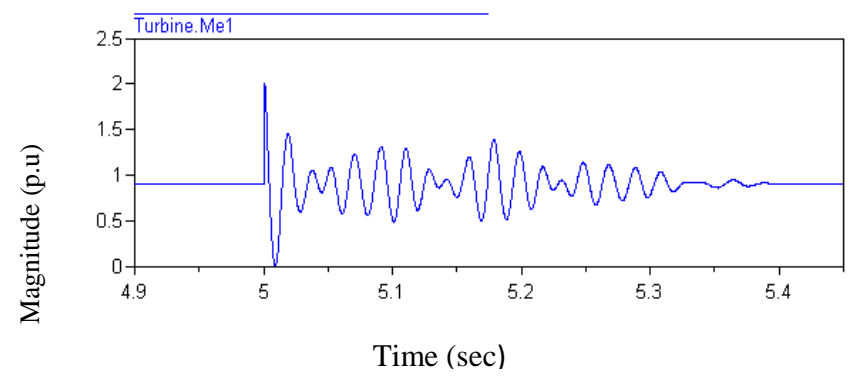

Figure 7c: Electromechanical Torque

\section{CONCLUTION}

In this paper discussed the problem of sub synchronous resonance and that the interaction between the frequencies in the electrical grid and the frequency of the turbine segments are equal at certain values if we change the value of the series capacitor. The first IEEE standard system was used for the purpose of studying the phenomenon of sub synchronous resonance. The system was analyzed by using eigenvalue the purpose of providing an explanation in case of stability and instability in this analysis. We have four unstable frequencies that lead to problems in the system therefore; different controllers are used for the purpose of improving the stability of the system in addition to damping the sub-synchronous resonance oscillation. This is done by using FACTS control. In this research one type of FACTS control are used Thyristor Control Series Compensation (TCSC) and also using Automatic Voltage Regulation (AVR). This research also discusses design optimal control Linear Quadratic Gaussain (LQG) by using three signals (generator terminal voltage, generator speed and the transmission line current) as a feedback comparing with reference points for the best control of the oscillations. We also notice the complexity of the system at full order stat $\left(26^{\text {th }}\right)$ and its difficulty in dealing with it so the work was done to reduce the roots of the system using the Square-Root Balanced Truncation (BALSQ) norm approximation technique is used in getting a reduced order model for controller design While maintaining a stable system. It also has the potential to control the oscillations of the system and enhance the stability of the system when a three phase short circit occurs.

\section{ACKNOWLEDGMENTS}

My thanks to all supportive people who gave me the advice and support during the work and writing of this paper. Thanks for each person who contributed in many ways to make this paper possible and to appear in its full form.

\section{REFERENCES}

[1] Anderson, P. and Farmer, R. 1996. Series Compensation of Power Systems. PBLSH, Encinitas.

[2] Anderson, P. Agrawal, B. and Ness, J.1989. Sub synchronous Resonance in Power Systems. IEEE Press, New York.

[3] IEEE SSR Working Group .1985. Terms, Definitions and Symbols for Sub-Synchronous Oscillations. IEEE Transactions on Power Apparatus and Systems, PAS104, 1326-1334

[4] Kundur, P.1994. Power System Stability and Control. McGraw-Hill, Inc.

[5] IEEE SSR Task Force .1977. First Benchmark Model for Computer Simulation of Sub synchronous Resonance.
IEEE Transactions on Power Apparatus and Systems, PAS-96, 1565-1571.

[6] Bongiorno, M., Svensson, J. and Ängquist, L. 2008.Online Estimation of Sub-Synchronous Voltage Components in Power Systems. IEEE Transactions on Power Delivery, 23, 410-418.

[7] Pillai, G. N. Jovcic, D.2005. SSR analysis with a new analytic model of thyristor controlled series capacitor. In: 15th PSCC, Liege, 22-26 August 2005.

[8] Meikandasivam, S. Nema, R. Jain S. 2008.Behavioral study of TCSC device - A MATLAB /Simulink implementation. In: Proceedings of world academy of science, engineering and technology, vol. 35; November 2008. ISSN 2070-3740.

[9] Kalman, R.E., Bucy, R.S.1961.New results in linear filtering and prediction theory, ASME Trans. D-J. Basic Eng. pp. 83, 95-108.

[10] Kwakernaak, H. Sivan, R.1972. Linear Optimal Control Systems, Wiley-Interscience, New York.

[11] Fleming, F.H., Rishel R.W.1975. Deterministic and Stochastic Optimal Control, Springer, New York.

[12] Basin, M. Alvarez D.2008. Optimal LQG controller for linear stochastic systems with unknown parameters, Journal of the Franklin Institute 345, pp.: 293-302.

[13] Petersen, I. R. and Pota, H. R.2003. Min max LQG optimal control of a flexible beam, Control Engineering Practice, 11, pp.: 1273-1287

[14] Nadia, Y. Majid, D.2009.Sub synchronous Resonance Damping in Interconnected Power Systems. IEEE Transaction on Power Apparatus \& System.

[15] IEEE SSR Task Force.1977. First benchmark model for computer simulation of sub synchronous resonance. IEEE Trans. Power Apparat. Syst., vol. PAS-96, pp. 1565-1572, Sept./Oct.1977.

[16] A.E. Hammed, M. El Sadek.1984.Application of a thyristor controlled VAR compensator for damping oscillation of sub synchronous oscillation in power system. IEEE Trans., PAS, vol. 103, no. 1 pp. 198-212

[17] Mohammad, H., A. and Javed, S.2010. Effects of TCSC Parameters and Control Structure on Damping of SubSynchronous Resonance. 4th International power engineering and optimization, Malaysia June 2010.

[18] R. J. Piwko, C. A. Wegner S. J. Kinney, and J. D. Eden.1996.Sub synchronous resonance performance tests of the Slat thyristor-controlled series capacitor. IEEE Trans. Power Delivery, vol. 11, pp. 1112-1119.

[19] C.H. Won and K. Gunaratne. 2002. Performance study of lqg, mcv, and risk sensitive control methods for satellite structure control. In American Control Conference Proceedings, vol. 3, pp. 2481-2486 vol.3.

[20] K. Zhou and J. C. Doyle.1998. Essentials of Robust Control, Prentice-Hall.

[21] I.M. Jaimoukha and E. M. Kasenally.1997. Implicitly Restarted Krylov Subspace Methods for Stable Partial Realizations, SIAM J MATRIX ANAL A, 18 (3), pp. 633652, July 1997. 